LAWRENCE LIVERMORE NATIONAL LABORATORY

\title{
Airbreathing Acceleration Toward Earth Orbit
}

John C. Whitehead

\section{July 8, 2007}

$43^{\text {rd }}$ AIAA/ASME/SAE/ASEE

Joint Propulsion Conference and Exhibit

Cincinnati, $\mathrm{OH}$

Paper Number AIAA-2007-5837

AIAA = American Institute of Aeronautics and Astronautics

ASME = American Society of Mechanical Engineers

SAE = Society of Automotive Engineers

ASEE = American Society of Engineering Education 
This document was prepared as an account of work sponsored by an agency of the United States Government. Neither the United States Government nor the University of California nor any of their employees, makes any warranty, express or implied, or assumes any legal liability or responsibility for the accuracy, completeness, or usefulness of any information, apparatus, product, or process disclosed, or represents that its use would not infringe privately owned rights. Reference herein to any specific commercial product, process, or service by trade name, trademark, manufacturer, or otherwise, does not necessarily constitute or imply its endorsement, recommendation, or favoring by the United States Government or the University of California. The views and opinions of authors expressed herein do not necessarily state or reflect those of the United States Government or the University of California, and shall not be used for advertising or product endorsement purposes. 


\author{
John C. Whitehead ${ }^{*}$ \\ Lawrence Livermore National Laboratory, Livermore, CA 94551
}

\begin{abstract}
As flight speed increases, aerodynamic drag rises more sharply than the availability of atmospheric oxygen. The ratio of oxygen mass flux to dynamic pressure cannot be improved by changing altitude. The maximum possible speed for airbreathing propulsion is limited by the ratio of air capture area to vehicle drag area, approximately Mach 6 at equal areas. Simulation of vehicle acceleration shows that the use of atmospheric oxygen offers a significant potential for minimizing onboard consumables at low speeds. These fundamental calculations indicate that a practical airbreathing launch vehicle would accelerate to near steady-state speed while consuming only onboard fuel, then transition to rocket propulsion. It is suggested that an aircraft carrying a rocket-propelled vehicle to approximately Mach 5 could be a realistic technical goal toward improving access to orbit.
\end{abstract}

\title{
I. Introduction
}

Considering the difficulty and cost of reaching earth orbit with rocket vehicles, the use of atmospheric oxygen for combustion (and additionally nitrogen as reaction mass) during very high-speed flight has been of widespread interest for decades. Generations of aerospace professionals and enthusiasts, along with entrepreneurs, military leaders, and pilots, have envisioned aircraft-like access to orbit while progress toward that end has been very slow.

This paper examines the physical limits to high-speed airbreathing flight. While many research results published over the decades have addressed pieces of the problem such as supersonic combustion and the thermal limits of materials, insight at the vehicle system level is offered here. The methodology is thought to be new, although it is acknowledged that the facts presented are inherent to much of the past work on the problem.

A goal is to offer an improved understanding of the big picture to aerospace decision-makers, and to technical specialists who focus on individual disciplines such as propulsion and high-speed aerodynamics. It is hoped that this paper will be useful to seasoned professionals who wish to gain perspective, as well as to students and young professionals who seek understanding for the first time.

Since the advent of computers and software that permit doing advanced analysis with relative ease, it has become common to perform complicated calculations of the type that require a myriad of assumptions, such as engine details, and vehicle design parameters including size and shape. A different approach taken here is to

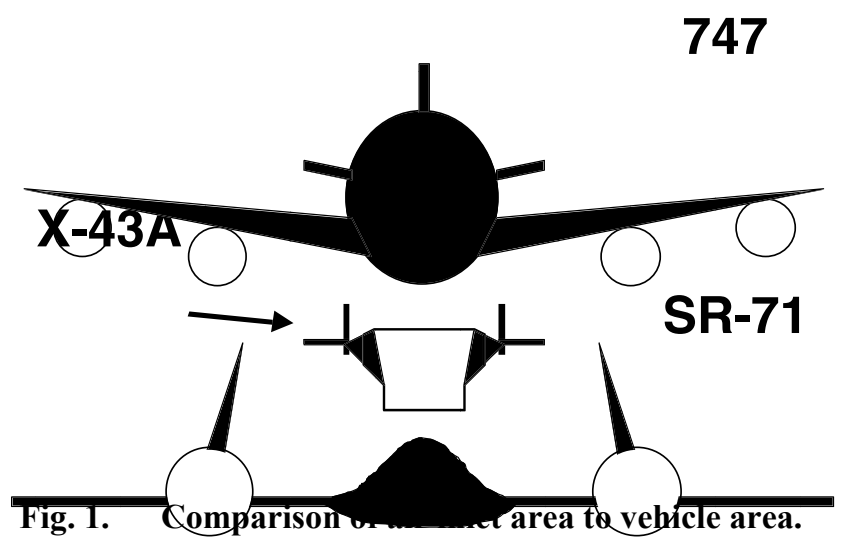

* PO Box 808, Mail Stop L-072, AIAA Senior Member. calculate, graph, and examine critically relevant information that is independent of uncertain or scopelimiting assumptions.

The most fundamental reason why aircraft can't fly arbitrarily fast is that aerodynamic drag rises as the square of velocity, while the power available from combustion, limited by oxygen flow, rises only linearly with velocity. The net effect is that faster flight requires the air capture area of engines to increase relative to vehicle drag area. Figure 1 illustrates this point by comparing frontal views of the Boeing 747, the Lockheed SR-71, and the NASA X-43A. The subsonic airliner needs only about $25 \%$ of its total frontal area to capture air, while the Mach 3 airplane uses about $40 \%$, compared to $80 \%$ in the Mach 10 test. 


\section{Oxygen Flux and Dynamic Pressure}

Above the speed of sound, the availability of oxygen is readily calculated as the product of air density, oxygen fraction, vehicle velocity, and the air capture area which feeds the engine(s). Normalizing to the latter quantity leads

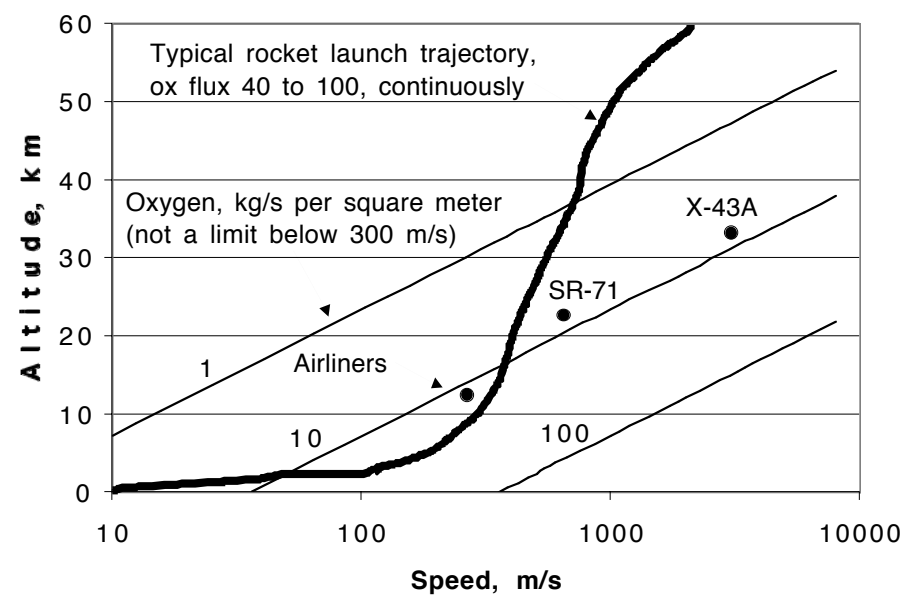

Fig. 2. Atmospheric oxygen availability is limited. to oxygen flux contours on a graph of altitude versus speed, Fig. 2. Air density decays exponentially from $1.225 \mathrm{~kg} / \mathrm{m}^{3}$ at sea level, with a scale height (1/e distance) of $7.0 \mathrm{~km}$. For practical purposes here, air is $23.3 \%$ oxygen by mass at all altitudes.

Figure 2 also shows a typical trajectory for a rocket-propelled launch vehicle. Rocket oxygen flow per square meter of vehicle cross sectional area is typically steady during ascent, e.g. just over $100 \mathrm{~kg} / \mathrm{s}-\mathrm{m}^{2}$ for the first stage of the Saturn $\mathrm{V},{ }^{1}$ and almost half that for the smallest liquid launch vehicles such as the SpaceX Falcon 1. Comparing the rocket oxygen flux with the atmospheric oxygen contours along its path makes it easy to appreciate that atmospheric oxygen is relatively scarce.

Airbreathing operating points, additionally plotted in Fig. 2, represent the three vehicles shown in Fig. 1. Their atmospheric oxygen fluxes are all near $10 \mathrm{~kg} / \mathrm{s}-\mathrm{m}^{2}$, only a tenth to a quarter of typical rocket values. It is clear that airbreathing flight toward orbit must somehow be very different from rocket trajectories.

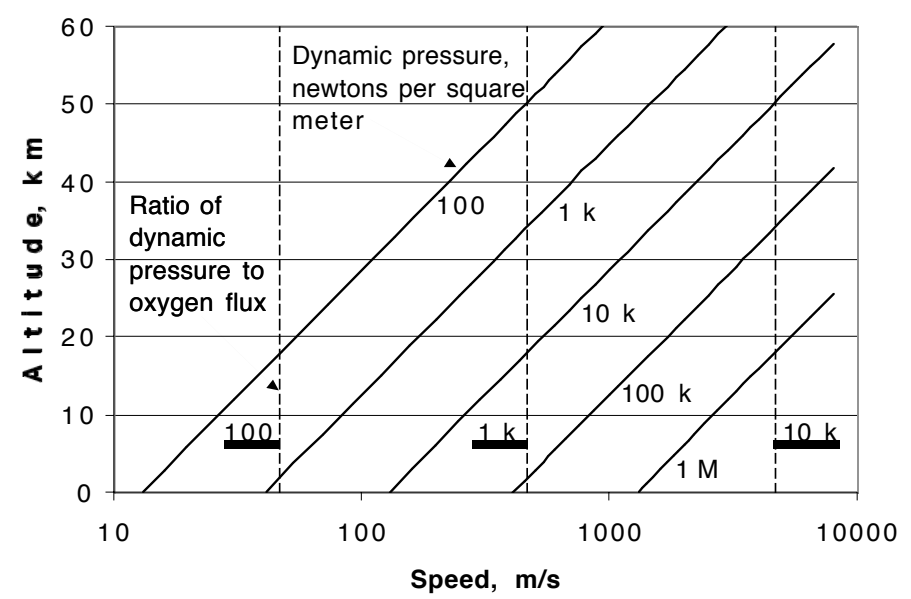

Fig. 3. Dynamic pressure contours on same axes as Fig. 2.

The reason why the rocket trajectory is so steep in Fig. 2 is of course to avoid atmospheric drag. While rocket vehicles climb early, an airbreathing launch vehicle would need to linger in the atmosphere while accelerating. Figure 3 shows dynamic pressure contours (solid lines) on the same axes as the Fig. 2 plot.

Consider the relative slopes of the contours in Figs. 2 and 3. Flying to higher speeds and altitudes at a constant atmospheric oxygen flux would require crossing the contours in Fig. 3, toward higher dynamic pressure and higher drag. Alternatively, accelerating at a constant dynamic pressure, parallel to the contours in Fig. 3, requires crossing the oxygen contours toward reduced availability of atmospheric oxygen.

The spacing of the contours along the horizontal axis in Fig. 2 is one per order of magnitude, because oxygen availability is linear with speed. In contrast, the solid lines in Fig. 3 are twice as close together along the speed axis, because dynamic pressure depends on the square of speed. Dynamic pressure is undesirable (drag) while oxygen flux is good (power), so it is appropriate to calculate the ratio of the two quantities. Both are linear in density (note equal vertical contour spacing in Figs 2 and 3). The quotient is simply speed divided by twice the atmospheric mass fraction of oxygen.

The vertical dashed contours in Fig. 3 represent the ratio of the quantities in Figs. 3 and 2, dynamic pressure to oxygen flux. While the ratio could be simply graphed as a function of speed, the contours emphasize the altitude independence. Visualizing any accelerating trajectory relative to the dashed lines makes it easy to appreciate that there is no way to fly above, below, or around an increasing amount of drag relative to airbreathing power.

While the foregoing information shows why there is a fundamental physical limit to the speed of airbreathing flight, a more useful comparison to consider is engine thrust relative to vehicle drag. 


\section{Energy, Power, Thrust, and Specific Impulse}

Rocket engines often run fuel rich, both to avoid extreme temperatures and to reduce the mean molecular weight of the exhaust. In contrast, propulsion in the air requires using the available reactants to heat a large amount of nitrogen. Even stoichiometry is assumed here, because it would be unwise to waste either oxygen or fuel. Heats of combustion are $58 \mathrm{kcal} / \mathrm{gram}-\mathrm{mole}$ for hydrogen (molecular weight 2), and $1300 \mathrm{kcal} / \mathrm{gram}$-mole for alkanes at molecular weight 114, assuming for both that the water resulting from combustion remains in the vapor phase.

Chemical energy from fuel combustion is converted into vehicle kinetic energy. On an instantaneous basis, the ideal loss-less situation (i.e. the physical limit case) is that the time rate of combustion energy is power applied to the vehicle, which equals thrust multiplied by speed. Combustion power depends on the mass flow of atmospheric oxygen, so speed can be eliminated from both sides of the equation to yield a simpler expression. An upper physical limit for supersonic thrust can be calculated as the product of oxygen density, air capture area, and combustion energy per unit mass of oxygen. The latter is $15.2 \mathrm{MJ} / \mathrm{kg}$ for hydrogen fuel, and $13.6 \mathrm{MJ} / \mathrm{kg}$ for hydrocarbons. It is notable that these numbers are close to one another, i.e. the fuel choice has only a small effect on the maximum possible thrust. Even though fuel mass flow differs greatly for the two cases, oxygen availability determines the

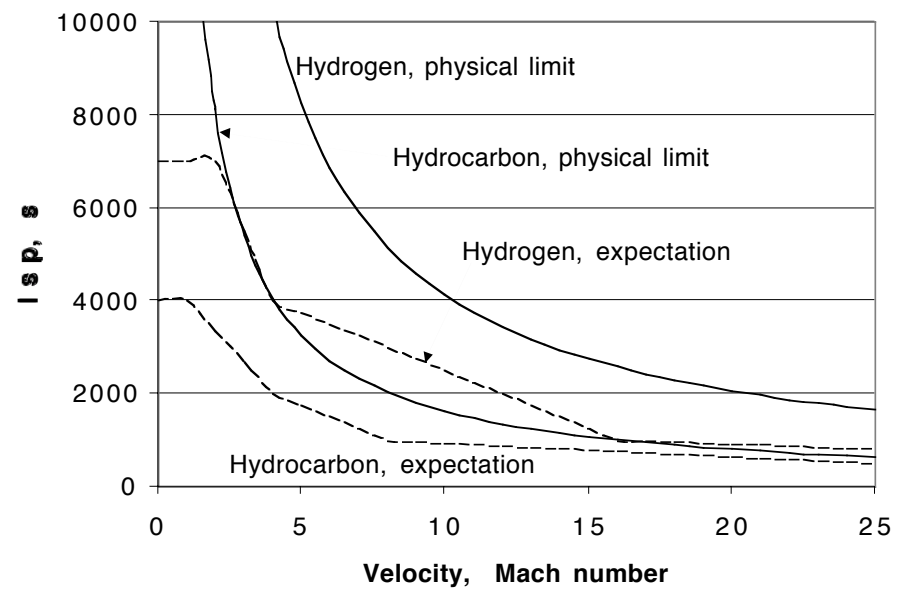

Fig. 4. Specific impulse used for airbreathing calculations. airbreathing power and the thrust limit.

As an example calculation using the above numbers, consider that the oxygen density at $30 \mathrm{~km}$ altitude is $0.004 \mathrm{~kg} / \mathrm{m}^{3}$. Multiplying by the energy yields 53 to $60 \mathrm{kN}$ thrust per square meter of air capture area. In reality, airbreathing engines cannot convert all the combustion energy into vehicle kinetic energy. There is a thermodynamic limit below the physical limit, and then actual capability below that. It is conventional to specify the relation between thrust and the flow of reactants in terms of fuel specific impulse, the duration over which an engine can produce thrust equal to the initial weight of the available fuel (i.e. not counting the oxygen). Figure 4 shows specific impulse curves from the literature (dashed lines). ${ }^{2,3}$ The solid curves in Fig. 4 represent the physical limit calculations described above, specifically combustion energy per unit fuel weight, divided by velocity.

While increasing the attainable specific impulse is a topic for further research such as airbreathing pulse detonation propulsion, ${ }^{4}$ it can be appreciated from Fig. 4 that there is an upper bound. Both sets of curves from Fig. 4 are used below, in order to illustrate the margin between technological expectations and physical limits.

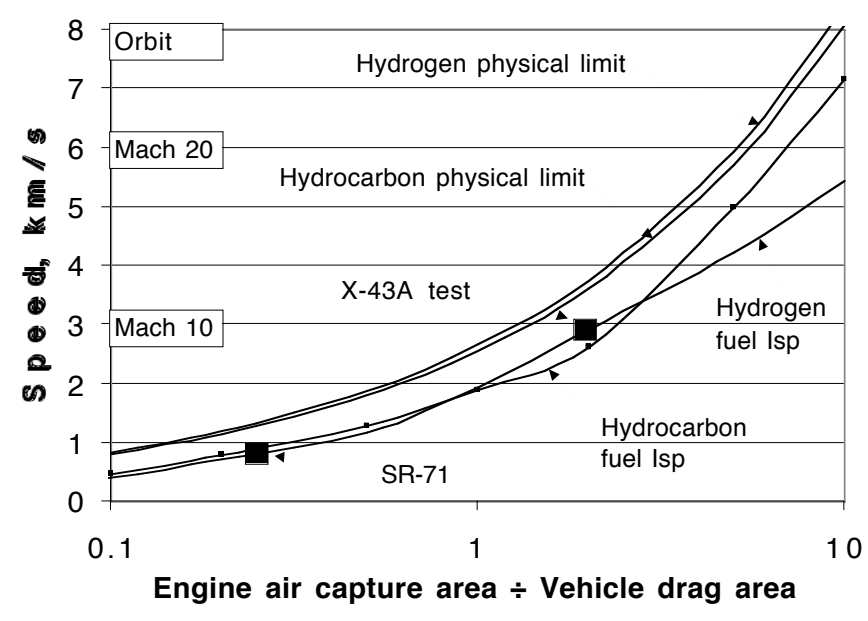

Fig. 5. Maximum flight speeds when thrust equals drag.

\section{Speeds Attainable with Drag}

Given the information presented in Figs. 2-4, it is straightforward to determine the limiting equilibrium speed of airbreathing vehicles, by setting thrust equal to drag. Assuming that the engines and airframe have no limitations related to combustion efficiency or material strength at temperature, the result is independent of air density and altitude for reasons noted above. Given that all captured oxygen is used, the only remaining variables are specific impulse, the air capture area of engines, and the vehicle's drag area $(\mathrm{CdA})$. Using the ratio of the latter two as a parameter avoids any need to restrict the result to a particular vehicle size.

Figure 5 shows curves for the two fuel options, using both the ideal loss-less energy 
cases and expectations for airbreathing fuel specific impulses. The curves are not dramatically different from one another, so fuel selection and further technology improvements would offer only modest gains beyond the expected values for $I_{S P}$. The left part of the graph is within the realm of existing technology, because engine inlet area can easily be less than vehicle drag area. For reference, the approximate operating points for the SR-71 and the X-43A are labeled based on their highest known speeds.

Per Fig. 5, achieving faster atmospheric flight requires reducing the drag coefficient while capturing air with most or all of a vehicle's area exposed to the oncoming air. Drag coefficients are typically less than unity, so it is possible for the air capture area to exceed the drag area, as the X-43A aptly demonstrated. ${ }^{5}$ Whether lower drag coefficients and greater air capture areas can be achieved experimentally remains unknown. In order for a purely airbreathing vehicle to reach orbital velocity beyond the right edge of the graph, the air capture area must exceed drag area by an order of magnitude.

The duration of flight and the rate of fuel consumption is a separate subject from the foregoing discussion of maximum possible flight speeds. The 747 airliner can cruise for 12 hours after taking off with a $44 \%$ fuel fraction, while the SR-71 needs to refuel every couple of hours at full speed despite its higher $55-60 \%$ fuel fraction. The experimental X-43A burned fuel for eleven seconds. Although sustaining constant speeds is not relevant to trajectories toward orbit, obtaining acceleration without undue fuel consumption is critically important.

\section{Fuel Consumption During Acceleration}

Simplifying assumptions, favoring airbreathing acceleration, are made here. Energy required to climb to higher altitudes is neglected, by merely ignoring gravity and lift forces. In addition, the drag coefficient is treated as a constant, whereas it typically is much higher in the transonic region. Another inherent assumption is unrestricted operating envelopes for airframes and engines. In particular all the available oxygen is assumed to combust fuel and generate full thrust aligned with the velocity vector over the entire speed range, in spite of potentially wide variations in thrust itself, dynamic pressure, specific impulse, altitude, angle of attack, temperature, etc.

In the absence of gravity, a vehicle's mass change due to airbreathing fuel consumption is a function of its velocity, regardless of thrust and time, just as is true for rockets. A differential equation in mass and velocity can be written and solved, using math somewhat more complicated than the rocket equation. There is a closed-form analytic solution for the physical limit case (thrust $=$ rate of combustion energy $\div$ speed), but not for the realistic situation in which fuel $\mathrm{I}_{\mathrm{SP}}$ is a non-simple function of speed. In both cases, there is a strong dependence on the ratio of engine air capture area to vehicle drag area, on the amount of chemical energy relative to fuel mass, and on the amount of oxygen required relative to fuel.

Figure 6 shows sets of curves for both fuels based on expected ISP per Fig. 4, at three values of the ratio of areas. For convenience, the curves were generated using numerical integration in the time domain. Velocity was calculated as the integral of thrust minus drag, divided by mass. Mass was calculated using its negative derivative that is

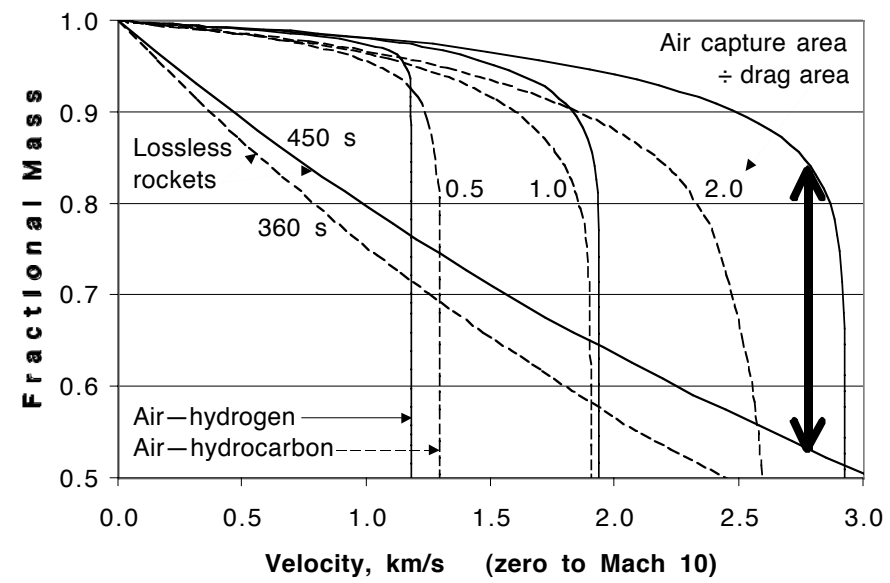

Fig. 6. Fuel consumed as a function of speed attained. proportional to air density, air capture area, and vehicle velocity.

Although physical values had to be chosen for the two areas, initial vehicle mass, and flight altitude, varying these parameters verified their unimportance to the curves shown (at constant ratios of areas). Time labels simply moved along the curves, e.g. slower acceleration for either heavier vehicles or higher altitudes at a chosen set of area values. Even large variations in altitude over the course of acceleration would not change Fig. 6.

The falling masses of rocket-propelled vehicles (no gravity or drag) are also plotted for comparison. A conclusive indication from Fig. 6 is that all the airbreathing options use much less consumable mass than rockets do, until speeds approach equilibrium. The airbreathing curves cross the rocket curves, because no further airbreathing acceleration is possible despite continued fuel consumption. As a check on the calculations, note that the vertical parts of the curves in Fig. 6 agree precisely with the lower two maximum flight speed curves in Fig. 5, for each corresponding fuel selection and ratio of areas. 
For the sake of clarity in Fig. 6, similarly shaped curves for the physical limit cases are omitted. Their vertical asymptotes would be positioned further to the right, as can be read from the upper pair of curves in Fig. 5. It is notable again that the physical limit assumption used here is so optimistic that it violates thermodynamics, i.e. zero energy in the exhaust, akin to a heat engine efficiency of 100 percent. At the lowest speeds, the physical limit assumption can be called optimistic in another respect-infinite $I_{S P}$ at zero speed (Fig. 4).

As would be expected, Fig. 6 shows that less hydrogen (solid curves) is consumed than hydrocarbon (dashed curves) during initial acceleration, for all 3 ratios of areas. However, hydrogen does not necessarily always have the higher maximum speed, which is determined by the air-to-fuel ratio in addition to specific impulse. At the highest speeds in Fig. 5, the hydrogen curve is the lowest, because it is difficult to obtain the higher oxygen flux needed to support hydrogen's 8-to-1 stoichiometric mixture ratio. Multiple crossings of the lower two curves in Fig. 5 are to some extent artifacts of the piecewise-linear $\mathrm{I}_{\mathrm{SP}}$ approximations in Fig. 4.

The large vertical double arrow near the right edge of Fig. 6 indicates the impressive mass margin that an airbreathing vehicle would have over a rocket vehicle in one particular case. If the extra mass needed for aircraft hardware (airframe, engines, landing gear, etc. compared to a similarly capable rocket stage) could be lighter than this $30 \%$ of gross launch mass, then a launch vehicle having an airbreathing first stage could deliver a greater payload starting with the same total mass. The results offer an even better outlook, to the extent that gross liftoff mass is not the best criterion for comparison. For instance, a larger and heavier airbreathing first stage could be practical if it is fully reusable over cost-effective repetitive flights.

\section{Conclusion}

The results shown in Figs. 5 and 6 are best-case limits that apply to all vehicles, regardless of size scale, engine design, flight altitude, airframe material strength, temperature tolerance, etc. Of course it is true that particular selections for these items could easily reduce the limits due to numerous practical considerations, but the fundamental limits would not be increased. Many of the practical considerations can be summed up by noting that rocket engines often operate at steady conditions over the course of accelerating their vehicles, but airbreathing engines cannot.

Considering the optimistic and nonrestrictive assumptions used for the calculations here, it is doubtful that it would ever be possible to fly to orbital speeds $(8 \mathrm{~km} / \mathrm{s})$ using airbreathing propulsion. Nevertheless, airbreathing first stages potentially have much to offer below their limiting speeds. The shape of the Fig. 6 curves shows that diminishing returns are reached abruptly, which suggests that the transition from airbreathing to rocket acceleration should be rapid, such as a staging event.

For a given vehicle size, larger air capture areas and lower hypersonic drag coefficients would extend the maximum possible speeds for airbreathing flight. Varying angle of attack would change drag coefficients, so the need to generate appropriate lift must be taken into account, along with all the other non-ideal effects that were neglected in this paper. An accurate estimate of drag for a practical airbreathing launch vehicle must include the presence of rocket upper stages and packaging for first stage fuel, in addition to one or more engines.

The present state of the art is that airplanes have carried small launch vehicles to almost Mach 1, and hypersonic engine testing has demonstrated Mach 10 flight while carrying very little fuel or payload. There is a range of potential options to explore and understand between these two extremes. One question worthy of future efforts is whether a practical airbreathing stage could reach roughly Mach 5 while carrying a rocket vehicle that is in turn capable of accelerating a payload the rest of the way to orbit.

\section{Acknowledgment}

This work was performed under the auspices of the U.S. Department of Energy, by the University of California, Lawrence Livermore National Laboratory under Contract W-7405-Eng-48.

\section{References}

\footnotetext{
${ }^{1}$ Whitehead, J.C., "Mass Breakdown of the Saturn V," AIAA Paper 2000-3141, July 2000.

2 Daines, R., and Segal, C., "Combined Rocket and Airbreathing Propulsion Systems for Space Launch Applications," Journal of Propulsion and Power, Vol. 14, No. 5, 1998, pp 605-612.

${ }^{3}$ Murthy, S.N.B., and Curran, E.T., High Speed Flight Propulsion Systems, Progress in Astro. \& Aero. Vol. 137, AIAA 1991.

${ }^{4}$ Ma, F., Choi, J., and Yang, V., "Propulsive Performance of Airbreathing Pulse Detonation Engines," Journal of Propulsion and Power, Vol. 22, No. 6, 2006, pp 1188-1203.

${ }^{5}$ Marshall, L.A., Bahm, C., Corpening, G.P., and Sherrill, R., "Overview with Results and Lessons Learned of the X-43A Mach 10 Flight," AIAA Paper 2005-3336, May 2005.
} 